\title{
Continuous wave OSL in bulk AIN single crystals
}

\author{
Alexander Vokhmintsev ${ }^{*}$, llya Weinstein, and Dmitriy Spiridonov \\ REC Nanomaterials and Nanotechnologies, Ural Federal University, Mira 19, 620002 Ekaterinburg, Russia
}

Received 22 July 2012, revised 1 November 2012, accepted 4 November 2012

Published online 21 December 2012

Keywords aluminum nitride, CW-OSL, oxygen-related centres, dose dependences

* Corresponding author: e-mail a.s.vokhmintsev@ustu.ru, Phone: +7 3433759374

The kinetics of recombination luminescence of $\beta$ irradiated AIN single crystals has been studied with continuous wave optically stimulated luminescence (CWOSL) method. It is shown that the OSL process is characterized by two exponential components with a decay time $\tau=32$ and $212 \mathrm{~s}$. Photoionization cross-sections $\sigma=$ $4.8 \cdot 10^{-19}$ and $7.3 \cdot 10^{-20} \mathrm{~cm}^{2}$ have been evaluated, assum-

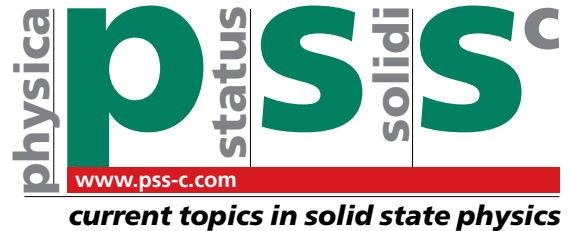

current topics in solid state physics ated, assum- $\quad$ state dosimetry.

ing each component is related to electron traps based on $\mathrm{V}_{\mathrm{N}^{-}}$and/or $\mathrm{O}_{\mathrm{N}^{-}}$-centers at various charged states. It is established that dose dependences of the OSL response of the bulk crystals under study are linear with taking into correct accounting for the radiation induced afterglow and hence can be used for quantitative estimates in solid
1 Introduction Single crystalline AIN is a semiconductor with the band gap about $6.2 \mathrm{eV}$. It is characterised by high temperature conductivity, its thermal expansion coefficient being close to that of silicon, thus ensuring its wide use in optoelectronics and microelectronics as UV emitter and UV detectors, substrate for hybrid integrated chip and power electronics [1-3]. Synthesis of first bulk AIN single crystals gave the fillip to a number of studies related to investigation of their emission properties with different spectroscopic methods [4-11]. Possible applications of the AlN single crystals in solid state dosimetry of $\beta$ - and UV-irradiation with methods of thermally and optically stimulated luminescence (TL and OSL) are shown in Ref. $[12,13]$. It is known that intrinsic and impurity defects of the anion sublattice $\left(\mathrm{V}_{\mathrm{N}^{-}}\right.$and $\mathrm{O}_{\mathrm{N}}$-centers $)$ determine emission activity of AlN in the UV/Vis spectral range and help to detect and store dosimetric information [1215]. Recently within the framework of formal kinetics analysis of the TL processes involving the above mentioned centers the values of the activation energy and effective frequency factor have been obtained [10, 13]; and dose dependences of the TL and OSL response have been analyzed [16, 17]. However, the basic parameters responsible for mechanisms of optically stimulated emission of oxygen-related complexes in bulk aluminum nitride have not been studied enough. In this context the present investigation was aimed at the study of kinetics of recombina- tion luminescence and quantitative estimation of microparameters of capture centers in $\beta$-irradiated AIN single crystals using continuous wave optically stimulated luminescence (CW-OSL).

2 Samples and methods Single crystalline samples of aluminum nitride (Nitride Crystals, Inc.) were obtained from industrial fine-dispersed AlN powder of semiconducting purity preliminary annealed by the sublimationrecondensation method in high vacuum at $1900-2000{ }^{\circ} \mathrm{C}$ in nitrogen atmosphere [18]. Single crystalline $\mathrm{SiC}$ (0001) substrates were used to synthesize the samples with the growth rate $40-55 \mu \mathrm{m} \cdot \mathrm{h}^{-1}$. According to the analysis data obtained by mass spectrometry with gas-discharge ionization, oxygen content in AIN does not exceed $10^{18} \mathrm{~cm}^{-3}$ [19]. The final samples had the form of disks $15 \mathrm{~mm}$ in diameter and $0.25-0.50 \mathrm{~mm}$ thick with epi-ready surfaces.

The CW-OSL and TL parameters were studied with custom-made spectrometric installation. The samples were irradiated by ${ }^{90} \mathrm{Sr} /{ }^{90} \mathrm{Y} \beta$-source with the dose rate of 32 $\mathrm{mGy} \cdot \mathrm{min}^{-1}$. Green DPSS laser $(150 \mathrm{~mW}, 532 \mathrm{~nm})$ was used for OSL measurements. Stimulating radiation intensity at the sample location was $25 \mathrm{~mW} \cdot \mathrm{cm}^{-2}$. The data were registered in the $3.25 \mathrm{eV}$ band with $\mathrm{FWHM}=0.27 \mathrm{eV}$ using a set of optical filters and a FEU-39A photomultiplier in counting regime. 
The samples were pre-irradiated with dose $D$ within the 2.7-960 mGy range. Then the AlN crystal was stored at room temperature for $5 \mathrm{~min}$ in darkness. Further the CW-OSL signal was registered for $10 \mathrm{~min}$ and the sample was annealed up to $700 \mathrm{~K}$. This thermal treatment is required for complete emptying of charge carrier traps responsible for luminescence within the registered spectral range. Then the measurements were repeated for different irradiation dose. For $D=64 \mathrm{mGy}$ the TL curves were registered before and after the CW-OSL measurement at heating rate $2 \mathrm{~K} \cdot \mathrm{s}^{-1}$.

3 Results Figure 1 shows the CW-OSL curves with varying dose of $\beta$-irradiation. It is seen that with increasing $D$ the initial intensity and lightsum increase within the dose range under study.

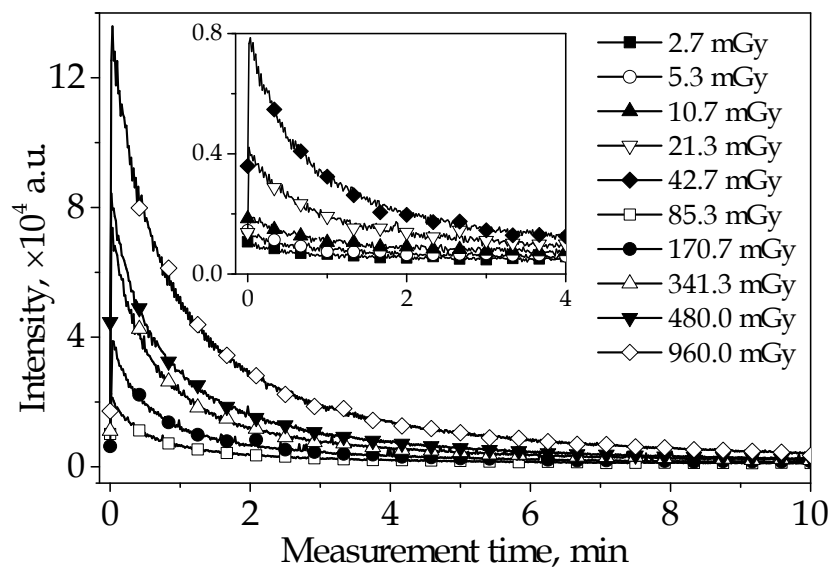

Figure 1 Experimental CW-OSL curves for $\beta$-irradiated AIN single crystal. Corresponding doses are shown in the figure.

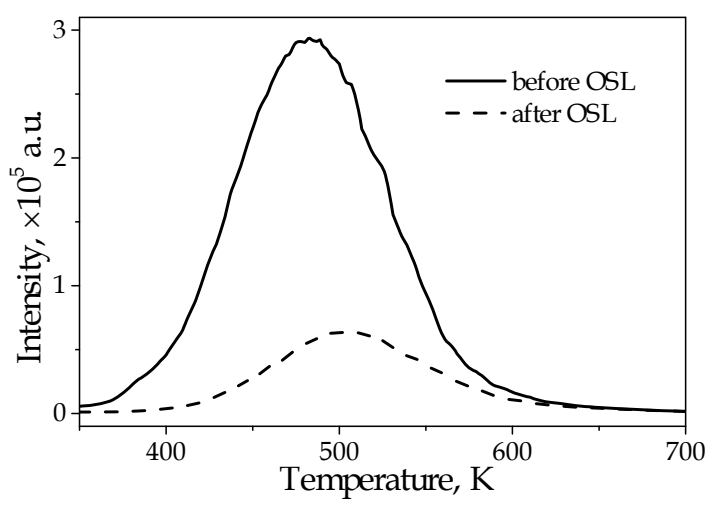

Figure 2 The TL curves for $\beta$-irradiated AIN single crystal.

Figure 2 demonstrates the measurement results of the TL response before and after OSL registration. Table 1 contains shape parameters of the TL peaks. It is seen that the TL curves look like a single wide unstructured peak, with maximum temperature $\left(T_{\max }\right)$, intensity $\left(I_{\max }\right)$ and lightsum $\left(S_{T L}\right)$ varied under optical stimulation. However, halfwidth $(\omega)$ and form-factor $\left(\mu_{g}\right)$ are practically un- changed. The value $\mu_{g}=0.50$ corresponds neither to the processes of the first- nor of the second order kinetics, thus pointing to complex behavior of the registered thermally activated glow [20].

Table 1 Shape parameters of TL.

\begin{tabular}{lccccc}
\hline \multicolumn{1}{c}{ parameter } & $\begin{array}{c}T_{\max }, \\
\pm 3 \mathrm{~K}\end{array}$ & $\begin{array}{c}\omega, \\
\pm 3 \mathrm{~K}\end{array}$ & $\begin{array}{c}\mu_{g} \\
\pm 0.01\end{array}$ & $\begin{array}{c}I_{\max }, \\
\times 10^{3} \text { a.u. }\end{array}$ & $\begin{array}{c}S_{\mathrm{TL}}, \\
\times 10^{6} \text { a.u. }\end{array}$ \\
\hline before OSL & 483 & 101 & 0.50 & 29.4 & 33.0 \\
after OSL & 506 & 104 & 0.50 & 6.4 & 7.6 \\
\hline
\end{tabular}

4 Discussion With this measurement technique the CW-OSL signal is overlapped by the sample afterglow (AG) at room temperature after $\beta$-irradiation (dashed line in Fig. 3). Note, that $A G$ is typical for different structures of the irradiated AlN and is present already before switching on the laser $[11,17]$. After initiation of stimulation ("laser on") the emission intensity increases dramatically and cumulative "OSL + AG" signal is registered (Fig. 3).

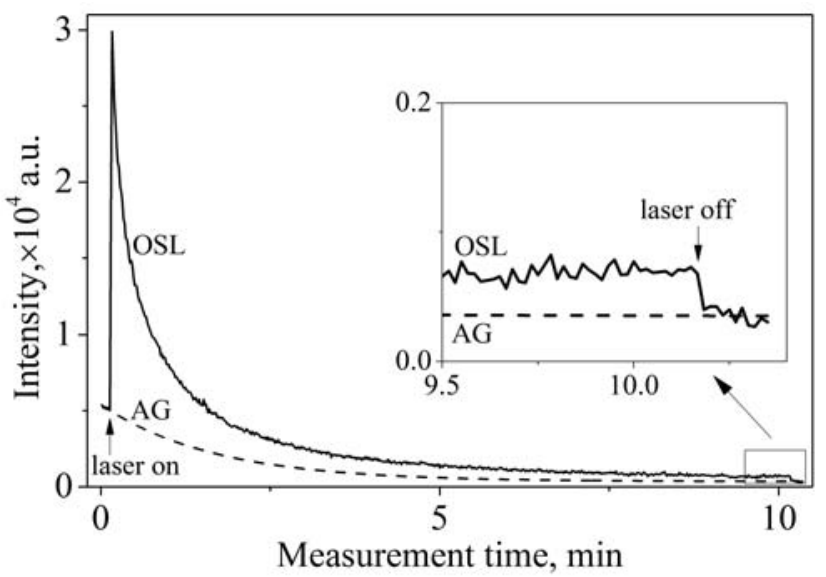

Figure 3 The CW-OSL (solid line) and AG (dashed line) curves for $\beta$-irradiated AlN single crystal with $D=128 \mathrm{mGy}$.

To analyze the CW-OSL kinetic features the effective signal was separated from the experimental curves (Fig. 1) by subtracting the AG curves. The latter were approximated by decay exponent. This mathematical approach is justified in Ref. [11], where AG of the irradiated AlN single crystals is shown to be described by exponential components with decay constants $59 \mathrm{~s}$ and $606 \mathrm{~s}$. Thus, $5 \mathrm{~min}$ after the end of irradiation (before laser stimulation) basic contribution to $\mathrm{AG}$ is made by a slow component.

Kinetic analysis of the experimental CW-OSL curves including AG was done in terms of the first order formalism [21]:

$$
I(t)=\sum_{i} I_{0 i} \exp \left(-\frac{t}{\tau_{i}}\right),
$$

where $I_{0 i}$ is the intensity at the initial moment of stimulation, a.u.; $\tau_{i}$ is the decay constant, $\mathrm{s} ; t$ is the measurement time (or optical stimulation), $\mathrm{s} ; i$ is the component number. 
Figure 4 shows approximation example of the OSL curve by Eq. (1) for $i=2$. Table 2 contains the parameter values of the CW-OSL curves calculated and $R^{2}$ determination coefficient. It is seen that all the OSL dependences are satisfactorily described by exponential components $\mathrm{C} 1$ and $\mathrm{C} 2$. This phenomenon can be explained by the presence of a number of optically active traps of charge carriers after $\beta$-irradiation, which are emptied at room temperature.

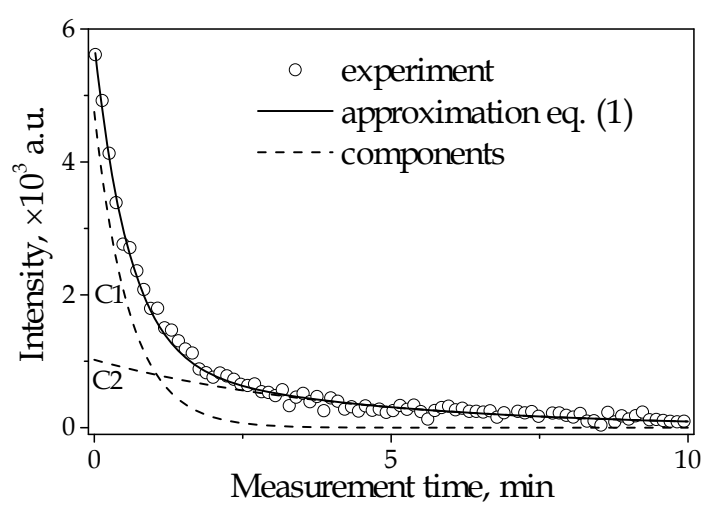

Figure 4 Example of the CW-OSL curve approximation by Eq. (1) for $\beta$-irradiated AIN single crystal with $D=42.7 \mathrm{mGy}$.

Table 2 Kinetic parameters of CW-OSL depending on the dose.

\begin{tabular}{cccccc}
\hline \multirow{2}{*}{$D, \mathrm{mGy}$} & \multicolumn{2}{c}{$\tau \pm 0.5, \mathrm{~s}$} & \multicolumn{2}{c}{$\sigma, 10^{-19} \mathrm{~cm}^{2}$} & \multirow{2}{*}{$R^{2}$} \\
\cline { 2 - 5 } & $\mathrm{C} 1$ & $\mathrm{C} 2$ & $\mathrm{C} 1$ & $\mathrm{C} 2$ & \\
\hline 2.7 & 19.4 & 30.3 & 7.7 & 4.9 & 0.871 \\
5.3 & 10.9 & 79.3 & 13.6 & 1.9 & 0.930 \\
10.7 & 30.9 & 146.3 & 4.8 & 1.0 & 0.960 \\
21.3 & 19.1 & 86.9 & 7.8 & 1.7 & 0.988 \\
42.7 & 35.3 & 250.3 & 4.2 & 6.0 & 0.994 \\
85.3 & 23.6 & 120.1 & 6.3 & 1.2 & 0.997 \\
170.7 & 28.5 & 208.5 & 5.2 & 0.7 & 0.996 \\
341.3 & 31.7 & 236.6 & 4.7 & 0.6 & 0.997 \\
480.0 & 33.1 & 223.7 & 4.5 & 0.7 & 0.998 \\
960.0 & 37.5 & 232.7 & 4.0 & 0.6 & 0.998 \\
\hline
\end{tabular}

Initial intensity $I_{0}$ of the OSL components increases with increasing irradiation dose within the range under study. This is in good agreement with the functional dependence $I_{0}=n_{0} / \tau$, where $n_{0}$ is the concentration of the occupied traps for charge carriers directly before optical stimulation [21]. Obviously, concentration $n_{0}$ must grow with increasing radiation dose. Note that upon variation of $D$ within the 42.7-960.0 mGy range the decay constants change slightly: $\tau=32 \pm 5$ and $212 \pm 43$ s for $\mathrm{C} 1$ and $\mathrm{C} 2$, respectively.

Assuming each decay component of the OSL response to be related to an independent trap, let us estimate photoionization cross-sections of the capture centers with Eq. (2) $[21]$ :

$$
\sigma=\frac{1}{\tau \Phi}
$$

where $\Phi=6.7 \times 10^{16} \mathrm{~cm}^{-2} \cdot \mathrm{s}^{-1}$ is stimulating radiation intensity.

Table 2 contains the obtained $\sigma$ values of the traps responsible for $\mathrm{C} 1$ and $\mathrm{C} 2$. For $D=42.7-960.0 \mathrm{mGy}$ the above photoionization cross-sections change slightly: $\sigma=$ $(4.8 \pm 0.7) \cdot 10^{-19}$ and $(7.3 \pm 1.5) \times 10^{-20} \mathrm{~cm}^{2}$ for $\mathrm{C} 1$ and $\mathrm{C} 2$, respectively.

Considering the shape parameters of the TL curves (Table 1) it can be concluded that, optical depletion of the charge carrier traps responsible for the forming lowtemperature part of peak dominates under laser stimulation of the irradiated sample. This is supported by the decrease of the lightsum and maximum intensity by 4.3 and 4.6 , respectively as well as by the shift of the maximum toward the high-temperature range by $23 \mathrm{~K}$. It is known that during storage of the AlN sample for 15 min in darkness the TL light sum decreases by 1.2 and the peak shifts by $5 \mathrm{~K}$ [11]. Similar changes of the TL peaks within the spectral range under study during optical stimulation and storage evidence that the same charge carrier traps take part in recombination OSL and AG processes of the irradiated bulk AlN single crystals. According to the analysis of independent experimental data one can assume that the OSL signal is caused at least by two types of traps which are thermally active within the RT- $600 \mathrm{~K}$ range. They may be the anion $\mathrm{V}_{\mathrm{N}^{-}}$and $\mathrm{O}_{\mathrm{N}^{-}}$-centers in different charged states, which form systems of allowed energy levels for electron capture in the irradiated AIN near the bottom of the conduction band.

Time measurements of the CW-OSL do not allow to assess the energy of defects levels in the forbidden band. However, it is known that the $\mathrm{O}_{\mathrm{N}}$-centers in different charge states form shallow electron traps [22], which are activated below RT and serve donors in the bleaching under study. In turn, the anion $\mathrm{V}_{\mathrm{N}}$-vacancies are deep donors with $0.5-0.9 \mathrm{eV}$ estimates range of activation energy [10, 13]. In this regard, it can be assumed that the observed decay components $\mathrm{C} 1$ and $\mathrm{C} 2$ characterize optically stimulated ionization of the donor $\mathrm{O}_{\mathrm{N}^{-}}$and $\mathrm{V}_{\mathrm{N}}$-centers, respectively.

The present study analyzes also the dose dependences of the light sum $(S)$ and maximum intensity $(I)$ of the OSL signal with and without taking into account the AG curves (Fig. 5). It is seen that the $S(D)$ curve with AG accounting is linear, the tangent slope being in two logarithmic coordinates $k=1.02 \pm 0.01$. The $S(D)$ curve without AG is essentially nonlinear: $k=0.87 \pm 0.01$. At the same time $k=$ $0.97 \pm 0.03$ for the $I(D)$ curve in both cases. Thus the OSL signal from $\beta$-irradiated AIN single crystals under study can be used to evaluate the absorbed dose.

AG affects essentially the CW-OSL dose dependences of the AIN powder and ceramics under UV-irradiation and the nonlinear dose dependence is assigned to the fading during irradiation $[16,17]$. Note that the observed afterglow is one of the channels for fading of dosimetric infor- 
mation. In this context the above data point to importance of accounting for AG when using the AIN single crystals as material for $\beta$-dosimetry with OSL method.

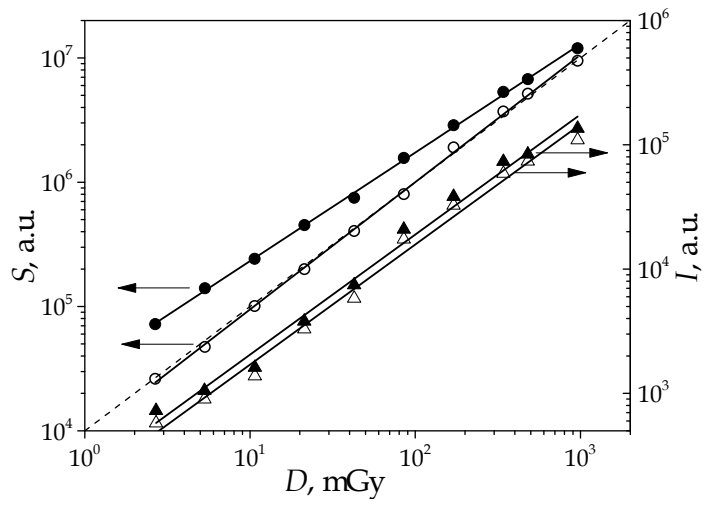

Figure $5 \mathrm{CW}-\mathrm{OSL}$ dose dependences for $\beta$-irradiated AlN single crystal with (open symbols) and without (solid symbols) taking into account the AG calculation. Solid lines - linear approximations; dashed line - ideal linear dependence $(k=1)$.

5 Conclusion Kinetic analysis of the OSL response of the irradiated AlN single crystals has been done within the framework of the first order kinetics formalism. It is shown that the luminescence process registered under optical stimulation of the samples after irradiation is characterized by fast and slow exponential components with the decay times $\tau=32$ and $212 \mathrm{~s}$, respectively. Photoionization cross-section of charge carrier traps $\sigma=4.8 \times 10^{-19}$ and $7.3 \times 10^{-20} \mathrm{~cm}^{2}$, responsible for $\mathrm{C} 1$ and $\mathrm{C} 2$ components, respectively, in the OSL signal, was evaluated. On the basis of the experimental data and analysis of independent data it is shown that various charged states of the $\mathrm{V}_{\mathrm{N}^{-}}$and $\mathrm{O}_{\mathrm{N}^{-}}$ centers may serve as electron traps participating in the recombination processes under study during optical or thermal stimulation of irradiated AIN single crystals. Optically active centers of capture are also responsible for the lowtemperature part of the TL peak at $480 \mathrm{~K}$. It is established that dose dependence of the OSL response in $\beta$-irradiated bulk AIN single crystals is linear with correct accounting for induced afterglow. Thus, the crystals under study can be used to detect $\beta$-irradiation and to solve the applied problems of OSL dosimetry.

Acknowledgements This work was fulfilled in terms of a State contract under the Federal Program «Scientific and Scientific-Pedagogical Staff of Innovational Russia 2009-2013».

\section{References}

[1] Y. Taniyasu, M. Kasu, and T. Makimoto, Nature 441, 325 (2006).

[2] A. BenMoussa, J. F. Hochedez, R. Dahal, J. Li, J. Y. Lin, H. X. Jiang, A. Soltani, J.-C. De Jaeger, U. Kroth, and M. Richter, Appl. Phys. Lett. 92, 022108 (2008).
[3] A. Teke and H. Morkoc, in: Springer Handbook of Electronic and Photonic Materials, edited by S. Kasap and P. Capper (Springer, NewYork, 2007), chap. 32.

[4] J. Senawiratne, M. Strassburg, N. Dietz, U. Haboeck, A. Hoffmann, V. Noveski, R. Dalmau, R. Schlesser, and Z. Sitar, Phys. Status Solidi C 2, 2774 (2005).

[5] S. M. Evans, N. C. Giles, L. E. Halliburton, G. A. Slack, S. B. Schujman, and L. J. Schowalter, Appl. Phys. Lett. 88, 062112 (2006).

[6] P. Lu, R. Collazo, R. F. Dalmau, G. Durkaya, N. Dietz, and Z. Sitar, Appl. Phys. Lett. 93, 131922 (2008).

[7] F. Tuomisto, J.-M. Maki, T. Yu. Chemekova, Yu. N. Makarov, O. V. Avdeev, E. N. Mokhov, A. S. Segal, M. G. Ramm, S. Davis, G. Huminic, H. Helava, M. Bickermann, and B. M. Epelbaum, J. Cryst. Growth 310, 3998 (2008).

[8] A. Y. Polyakov, N. B. Smirnov, A. V. Govorkov, T. G. Yugova, K. D. Scherbatchev, O. A. Avdeev, T. Yu. Chemekova, E. N. Mokhov, S. S. Nagalyuk, H. Helava, and Yu. N. Makarov, J. Phys. B 404, 4939 (2009).

[9] M. Bickermann, B. M. Epelbaum, O. Filip, P. Heimann, M. Feneberg, S. Nagata, and A. Winnacker, Phys. Status Solidi C 7, 1743 (2010).

[10] V. A. Soltamov, I. V. Ilyin, A. A. Soltamova, D. O. Tolmachev, E.N. Mokhov, and P. G. Baranov, Diam. Relat. Mater. 20, 1085 (2011)

[11] A. S. Vokhmintsev, I. A. Weinstein, and D. M. Spiridonov, J. Lumin. 132, 2109 (2012).

[12] L. Trinkler and B. Berzina, in: Advances in Ceramics Characterization, Raw Materials, Processing, Properties, Degradation and Healing, edited by C. Sikalidis (InTech, City, 2011), chap. 4.

[13] I. A. Weinstein, A. S. Vokhmintsev, and D. M. Spiridonov, Diam. Relat. Mater. 25, 59 (2012).

[14] T. Mattila and R. M. Nieminen, Phys. Rev. B 54, 16776 (1996).

[15] T. Mattila and R. M. Nieminen, Phys. Rev. B 55, 9571 (1997).

[16] L. Trinkler, L. Bøtter-Jensen, P. Christensen, and B. Berzina, Radiat. Prot. Dosim. 92, 299 (2000).

[17] A. S. Vokhmintsev, I. A. Weinstein, D. M. Spiridonov, D. A. Beketov, and A. R. Beketov, Tech. Phys. Lett. 38, 160 (2012).

[18] E. N. Mokhov, O. V. Avdeev, I. S. Barash, T. Yu. Chemekova, A. D. Roenkov, A. S. Segal, A. A. Wolfson, Yu. N. Makarov, M. G. Ramm, and H. Helava, J. Cryst. Growth 281, 93 (2005)

[19] J.-M. Maki, I. Makkonen, F. Tuomisto, A. Karjalainen, S. Suihkonen, J. Raisanen, T. Yu. Chemekova, and Yu. N. Makarov, Phys. Rev. B 84, 081204 (2011).

[20] R. Chen and S.W.S. McKeever, Theory of Thermoluminescence and Related Phenomena (World Scientific, Singapore, 1997), p. 576.

[21] L. Botter-Jensen, S.W.S. McKeever, and A.G. Wintle, Optically Stimulated Luminescense Dosimetry (Elsevier, New York, 2003), p. 355.

[22] V. A. Soltamov, I. V. Ilyin, A. A. Soltamova, D. O. Tolmachev, N. G. Romanov, A. S. Gurin, E. N. Mokhov, and P. G. Baranov, Phys. Status Solidi C 9, 745 (2012). 